\title{
Clinical utility of measuring expression levels of KAP1, TIMP1 and STC2 in peripheral blood of patients with gastric cancer
}

\author{
Yuan-Yu Wang ${ }^{1}$, Li Li ${ }^{2}$, Zhong-Sheng Zhao ${ }^{2 *}$ and Hui-Ju Wang ${ }^{3}$
}

\begin{abstract}
Background: We examined preoperative kinesin II-associated protein (KAP1), TIMP metallopeptidase inhibitor 1 (TIMP1) and stanniocalcin 2 (STC2) expression levels in patients with gastric cancers to assess their clinical application for diagnosing and monitoring diseases.

Methods: Real-time reverse transcription-polymerase chain reaction was used to detect the expression levels of KAP1, TIMP1, STC2, talin 2 (TLN2), sushi-repeat-containing protein, X-linked 2 (SRPX2) and secreted protein, acidic, cysteine-rich (SPARC) in the patients' peripheral blood karyocytes. The data were analyzed with receiver operating characteristics (ROC) curves.

Results: A total of 112 patients with gastric cancer, 42 patients with recurrence and 107 healthy volunteers were recruited. There were significant correlations between KAP1, TIMP1 and STC2 levels, and TNM tumor stages and distant metastases. The area under the ROC curves (AUC) of KAP1 was $0.803 \pm 0.040(P=0.0001)$, the AUC of TIMP1 was $0.767 \pm 0.043(P=0.0001)$ and the AUC of STC2 was $0.769 \pm 0.045(P=0.0001)$, thus differentiating preoperative gastric cancer patients from healthy volunteers by ROC curve analysis. The AUC of STC2 was $0.739 \pm 0.070(P=$ $0.004)$ and the AUC of KAP1 was $0.418 \pm 0.088(P=0.319)$, thus differentiating recurrence of gastric cancer from healthy volunteers by ROC curve analysis. High TIMP1 and STC2 expression levels were suspected to be poor prognostic factors of disease recurrence in patients with gastric cancer.
\end{abstract}

Conclusions: KAP1, TIMP1 and STC2 expression levels may be potential biomarkers for the screening, diagnosis, prognosis and surveillance of gastric cancer.

Keywords: Gastric cancer, Kinesin II-associated protein, TIMP metallopeptidase inhibitor 1, Stanniocalcin 2

\section{Background}

The overall 5-year survival rate in China for patients with gastric cancer is low, at $40 \%$, and the rate of lymph node metastasis is higher (50 to $75 \%$ ) [1]. Patients who had undergone potentially curative surgery retain the risk of recurrence mainly because of tumor dissemination via the blood or lymphatic circulations. To improve the cure rates for patients with gastric cancer, the primary tumors must be detected at an early stage, and recurrent disease must be diagnosed while it is still minimal or clinically occult; micrometastases are currently

\footnotetext{
* Correspondence: zhaozhongsheng1950@163.com

${ }^{2}$ Department of Pathology, Zhejiang Provincial People's Hospital, 158

shangtang road, Hangzhou, Zhejiang, PR China310014

Full list of author information is available at the end of the article
}

undetectable by conventional methods [2,3]. Conventional serum markers, however, lack sufficient sensitivity and specificity to facilitate early detection of cancer. To improve the poor survival outcome and to permit earlier diagnosis, there is a need for new and more sensitive biomarkers than those currently available, such as carcinoembryonic antigen and CA19-9 [4].

TIMP metallopeptidase inhibitor 1 (TIMP1) belongs to the tissue inhibitor of metalloproteases (TIMP) gene family. The proteins encoded by this gene family are natural inhibitors of the matrix metalloproteinases (MMPs), a group of peptidases involved in degradation of the extracellular matrix. In addition to its inhibitory role against most of the known MMPs, the encoded protein is also able to promote cell proliferation in a wide range

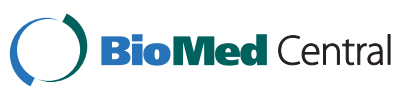


of cell types, and may also have an anti-apoptotic function. TIMP1 has been shown to be overexpressed in both liver and peritoneal metastases from patients with colorectal adenocarcinoma [5] and malignant thyroid neoplasms [6].

Stanniocalcin 2 (STC2) encodes a secreted, homodimeric glycoprotein that is expressed in a wide variety of tissues and may have autocrine or paracrine functions. The protein may play a role in the regulation of renal and intestinal calcium and phosphate transport, cell metabolism, or cellular calcium/phosphate homeostasis. Constitutive overexpression of human STC2 in mice resulted in preand postnatal growth restriction, reduced bone and skeletal muscle growth, and organomegaly. Expression of this gene is induced by estrogen and altered in some breast cancers [7]. The expression of STC2 was significantly correlated with lymph node metastasis, lymphatic invasion, and distant metastasis of esophageal squamous cell cancer [8] and colorectal cancer [9].

The protein encoded by $K A P 1$ mediates transcriptional control by interacting with the Kruppel-associated box repression domain found in many transcription factors. The protein, a member of the tripartite motif family, localizes to the nucleus and is thought to associate with specific chromatin regions. This tripartite motif includes three zinc-binding domains, a RING, a B-box type 1 and a B-box type 2, and a coiled-coil region. KAP1 plays an important role in progression to peritoneal carcinomatosis in gastric cancer patients [10].

In our previously study, KAP1, TIMP1, STC2, talin 2 (TLN2), sushi-repeat-containing protein, X-linked 2 (SRPX2), ITGB1 and secreted protein, acidic, cysteine-rich $(S P A R C)$ were selected by the Affymetrix GeneChip ${ }^{\text {TM }}$ HGU133A2.0 array, and were upregulated (ratio $\geq 2$ ) in gastric tumor tissue [11] (Affymetrix, Santa Clara, California, United States). In this study, we detected the expression of KAP1, TIMP1, STC2, TLN2, SRPX2, integrin beta 1 (ITGB1) and SPARC mRNAs in peripheral blood samples from pre-operative gastric cancer patients, patients with recurrence, and healthy volunteers. We compared the relationships between these results and clinical findings to assess the diagnostic value of the biomarkers in these patients.

\section{Methods}

\section{Patients and peripheral blood samples}

A total of 112 patients with gastric cancer and 42 patients with recurrence, diagnosed and treated between January 2006 and December 2010 at Zhejiang Provincial People's Hospital, were enrolled in this study. The median age of the gastric cancer patients was 60 years (range, 36 to 87 years), and the study comprised 77 males and 35 females. The median age of patients with recurrence was 64 years (range, 51 to 65 years), and this subset comprised 31 males and 11 females. One hundred and seven healthy volunteers who visited the hospital for a health examination and who had normal appearance of the gastric mucosa on gastroscopic examination were also enrolled. The healthy controls comprised 76 males and 31 females, with a median age of 59 years (range, 35 to 86 years).

All patients had follow-up records from the date of their operation, with a follow-up deadline of November 2011. None of the 112 patients with gastric cancer had received preoperative chemotherapy or radiotherapy, but were receiving radical or palliative gastrectomy. Seventy of these patients $(62.5 \%)$ were resected with tumor-free resection margins (R0), while the remaining 42 patients (37.5\%) were resected with R1 or R2. Following surgery, patients with stage I to III tumors received adjuvant chemotherapy, while patients with stage IV tumors received systemic chemotherapy with various combinations of regimens. Ten patients with recurrence received a radical operation again, and all received systemic chemotherapy. Of the 112 gastric patients with cancer, $12.5 \%$ (14 of 112 ) were classified as stage I, 35.71\% (40 of 112) were stage II, 34.82\% (39 of 112) were stage III, and $16.96 \%$ (19 of 112) were stage IV (Table 1). The clinicopathological findings were determined according to classification of malignant tumors as set out by the World Health Organization for gastric cancer. The study was approved and monitored by the ethics committee of our hospitals, and informed consent was obtained from each patient and healthy volunteer.

\section{Total RNA preparation}

A 2-ml sample of peripheral blood from all of subjects was collected in test tubes containing sodium citrate.

\section{Table 1 The characterization of the patient groups}

\begin{tabular}{|c|c|c|c|}
\hline & $\begin{array}{c}\text { Healthy } \\
\text { volunteers }\end{array}$ & $\begin{array}{l}\text { Gastric } \\
\text { cancer }\end{array}$ & $\begin{array}{c}\text { Recurrent } \\
\text { patients }\end{array}$ \\
\hline Age (range) & 59 y $(35$ to $86 y)$ & 60 y (36 to 87 y) & 64 y $(51$ to 65 y) \\
\hline \multicolumn{4}{|l|}{ Gender } \\
\hline Female & 31 & 35 & 11 \\
\hline Male & 76 & 77 & 31 \\
\hline \multicolumn{4}{|l|}{ TNM Stage } \\
\hline 1 & & 14 & 0 \\
\hline$\|$ & & 40 & 13 \\
\hline III & & 39 & 26 \\
\hline IV & & 19 & 3 \\
\hline \multicolumn{4}{|l|}{$\begin{array}{l}\text { Resection } \\
\text { margins }\end{array}$} \\
\hline $\mathrm{RO}$ & & 70 & 42 \\
\hline $\mathrm{R} 1$ & & 17 & \\
\hline $\mathrm{R} 2$ & & 23 & \\
\hline
\end{tabular}


Total RNA from peripheral blood karyocyte was isolated using QIAamp RNA Blood Mini Kits (Cat. No: 52304, QIAGEN, Hilden, Germany) following the manufacturer's protocol.

\section{Real-time quantitative PCR}

The expressions of KAP1, TIMP1, STC2, TLN2, SRPX2 and $S P A R C$ in peripheral blood from 82 patients with gastric cancer, 24 patients with recurrence and 69 healthy volunteers were confirmed by RT-PCR(reverse transcription PCR). Total RNA was extracted and cDNAs were reverse-transcribed by RevertAid ${ }^{\mathrm{TM}}$ reverse transcriptase. Real-time PCR was carried out using the ABI PRISM 7700 Sequence Detection System (Life Technologies, Grand Island, New York, United States) at $50^{\circ} \mathrm{C}$ for $2 \mathrm{~min}, 95^{\circ} \mathrm{C}$ for $10 \mathrm{~min}$, followed by 50 cycles at $95^{\circ} \mathrm{C}$ for $15 \mathrm{~s}$, and $60^{\circ} \mathrm{C}$ for $1 \mathrm{~min}$. The primers are listed in Table 2. The expression of GAPDH was used to normalize that of the target genes. Each assay was done in triplicate, the average was calculated, and the expression level of targets mRNA was expressed as $2^{-\Delta \mathrm{Ct}}, \Delta C t=C t$ (targets $m R N A)-C t(G A P D H)$.

\section{Statistical analysis}

Statistical analysis was performed using SPSS 16.0 (SPSS Inc, Chicago, United States). The level of significance was set at $P<0.05$. ROC (receiver operation characteristics) curves were constructed by calculating the sensitivities and specificities of a biomarker or the diagnostic score of a logistical regression model at different cut-off points for differentiating gastric cancer cases from healthy volunteers. The area under the ROC curves (AUC) was statistically interpreted as the probability to correctly distinguish patients with gastric cancer from normal subjects. An area of 1.0 represented a perfect test, and an area of 0.5 represented a worthless test. The $P$ value was the probability that the sample AUC was found when, in fact, the true (population) AUC was 0.5 (null hypothesis: area $=0.5)$. If $P$ was low $(P<0.05)$, then it was concluded that the AUC was significantly different between the two groups. The ROC curves were used to determine the optimal cut-off values (with the Youden J test for overall accuracy) [12]. The cutoff value corresponded to the optimal diagnostic accuracy (the highest sum of the respective values for sensitivity and specificity) [6].

\section{Results}

The diagnostic efficacy of KAP1, TIMP1, STC2, TLN2, SRPX2 and SPARC in patients with gastric cancer

To evaluate the diagnostic value of KAP1, TIMP1, STC2, $T L N 2, S R P X 2, I T G B 1$ and SPARC expression levels in the diagnosis of patients with gastric cancer, the AUC value from ROC curve analysis was determined (Figure 1). The clinical values were assessed by differentiating preoperative gastric cancer patients from healthy volunteers. The AUC of KAP1 was $0.803 \pm 0.040(P=0.0001 ; 95 \%$ CI 0.724 to 0.881 ), the criterion value (cutoff value) was 0.0386 with a sensitivity of $76.9 \%$, and the specificity was $76.6 \%$. The AUC of TIMP1 was $0.767 \pm 0.043(P=0.0001 ; 95 \% \mathrm{CI}$ 0.682 to 0.851 ), the criterion value (cutoff value) was 0.215 with a sensitivity of $61.5 \%$, and the specificity was $83.0 \%$. The AUC of STC2 was $0.769 \pm 0.045(P=0.0001 ; 95 \% \mathrm{CI}$ 0.680 to 0.858 ), the criterion value (cutoff value) was 0.01 with a sensitivity of $94.9 \%$, and the specificity was $53.2 \%$. The AUC of SRPX2 was $0.507 \pm 0.059(P=0.901 ; 95 \% \mathrm{CI}$ 0.392 to 0.621$)$. The AUC of SPARC was $0.452 \pm 0.055$ $(P=0.372 ; 95 \%$ CI 0.345 to 0.559$)$. The AUC of TLN2 was $0.485 \pm 0.056(P=0.783 ; 95 \%$ CI 0.376 to 0.594$)$.

\section{Expression of KAP1, TIMP1 and STC2 in patients with gastric cancer and healthy volunteers}

According to the criterion value (cutoff value), the higher expression of KAP1 was detected in 21 of 107 (19.6\%) healthy volunteers and in 99 of 112 (88.4\%) patients with gastric cancer $\left(X^{2}=104.5, P=0.0001\right)$ (Figure 2$)$. The higher expression of TIMP1 was detected in 19 of 107 (17.8\%) healthy volunteers and in 100 of 112 (89.3\%) patients with gastric cancer $\left(X^{2}=112.8, P=0.0001\right)$. The higher expression of STC2 was detected in 20 of 107 (18.7\%) healthy volunteers, and in 101 of 112 (90.2\%) patients with gastric cancer $\left(\chi^{2}=113.1, P=0.0001\right)$.

Table 2 Primers used in real-time quantitative PCR

\begin{tabular}{llll}
\hline Gene & $\mathbf{F}$ & $\mathbf{R}$ & Lengths \\
\hline KAP1 & GAAGGCTATGGCTTTGGGTC & CAGGCGTTCAAGGCTCACT & 165 bp \\
TIMP1 & CCTGTTGTGGTGTGGTGAT & GGTTGTGGGACCTGTGGAAGTA & 272 bp \\
STC2 & CATCCTATCCGTCAACTCATCAG & TACGCTTGGTTCTTGGTGTCT & 71 bp \\
TALIN1 & TGAAGCGAGCCTCAGATAAT & CACCACCACTGTCTCATTCTC & 83 bp \\
SRPX2 & CGCTGCCCAACTCTGAAACCTC & ACCACTGGCGGCTGGACTGA & 151 bp \\
SPARC & TTCTCACATAAGCCCAGTTCA & GAACAACCGATTCACCAACTC & 406 bp \\
GAPDH & TGAAGGTCGGAGTCAACGG & CTGGAAGATGGTGATGGGAT & 195 bp \\
\hline
\end{tabular}




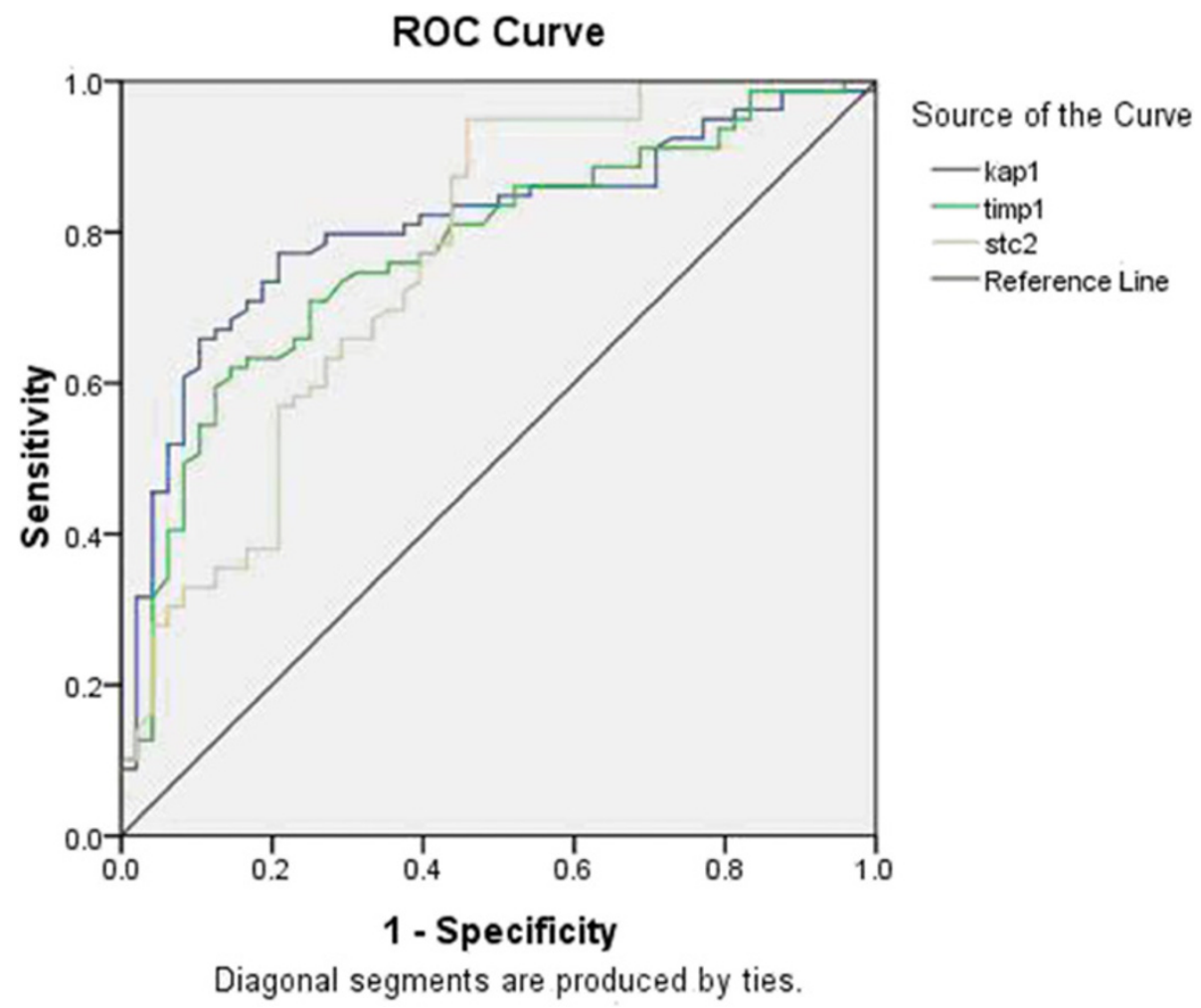

Figure 1 Receiver operation characteristics (ROC) curve of KAP1, TIMP1 and STC2 in peripheral blood of patients with gastric cancer and healthy volunteers. The clinical values were assessed by differentiating 112 preoperative gastric cancer patients from 107

healthy volunteers.

Relationship between expression of KAP1, TIMP1 and STC2 in patients with gastric cancer and clinical characteristics We examined the correlation between the expression levels of KAP1, TIMP1 and STC2 and clinical parameters. No significant associations were found between these three mRNAs, and gender, tumor location, tumor size, Lauren classification, histology classification, differentiation or distant metastasis $(P>0.05)$. There were, however, significant association between the three mRNAs and TNM stages and lymph node metastasis (Table 3 ).

The diagnostic efficacy of KAP1, TIMP1, STC2, TLN2, SRPX2 and SPARC in patients with recurrence of gastric cancer To evaluate the diagnostic value of KAP1, TIMP1, STC2, TLN2, SRPX2 and SPARC expression levels in the diagnosis of patients with recurrence of gastric cancer, the AUC value from ROC curve analysis was determined (Figure 3). The AUC of TIMP1 was $0.761 \pm$ $0.073(P=0.002 ; 95 \%$ CI 0.619 to 0.903$)$, the criterion value (cutoff value) was 0.214 with a sensitivity of $64.7 \%$, and the specificity was $83.0 \%$. The AUC of STC2 was $0.739 \pm 0.070 \quad(P=0.004 ; 95 \%$ CI 0.603 to 0.875 ), the criterion value (cutoff value) was 0.0129 with a sensitivity of $94.1 \%$, and the specificity was $55.3 \%$. The AUC of KAP1 was $0.418 \pm 0.088(P=0.319$; $95 \%$ CI 0.246 to 0.59$)$. The AUC of TLN2 was $0.441 \pm$ $0.083(P=0.475 ; 95 \% \mathrm{CI} 0.279$ to 0.603$)$. The AUC of SRPX2 was $0.453 \pm 0.074(P=0.569 ; 95 \%$ CI 0.308 to 0.599). The AUC of SPARC was $0.416 \pm 0.080 \quad(P=$ 0.308 ; $95 \%$ CI 0.26 to 0.573$)$.

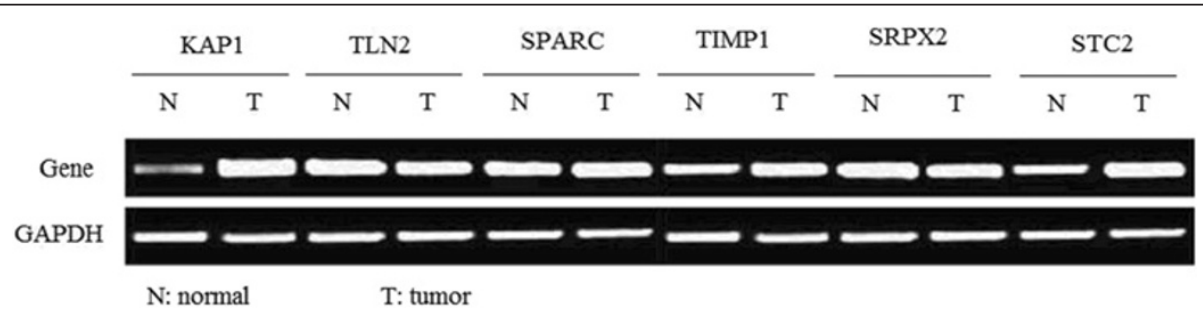

Figure 2 The gel electrophoresis of KAP1, TIMP1, STC2, TLN2, SRPX2, and SPARC in patients with gastric cancer and healthy volunteers. 
Table 3 Relationship of KAP1, TIMP1 and STC2 expression with pathological parameters of tumor

\begin{tabular}{|c|c|c|c|c|c|c|c|c|c|c|c|c|}
\hline \multirow[t]{2}{*}{ Clinical parameters } & \multicolumn{4}{|c|}{ KAP1 } & \multicolumn{4}{|c|}{ TIMP1 } & \multicolumn{4}{|c|}{ STC2 } \\
\hline & Low & High & $x^{2}$ & $P$ & Low & High & $x^{2}$ & $\mathbf{P}$ & Low & High & $x^{2}$ & $P$ \\
\hline $\mathrm{N}$ & 13 & 99 & & & 12 & 100 & & & 11 & 101 & & \\
\hline Gender & & & 1.52 & 0.22 & & & 0.68 & 0.41 & & & 3.08 & 0.079 \\
\hline Male & 7 & 70 & & & 7 & 70 & & & 5 & 72 & & \\
\hline Female & 6 & 29 & & & 5 & 30 & & & 6 & 29 & & \\
\hline Location & & & 0.184 & 0.91 & & & 1.82 & 0.40 & & & 1.99 & 0.369 \\
\hline Proximal & 1 & 11 & & & 0 & 12 & & & 0 & 12 & & \\
\hline Middle & 4 & 32 & & & 5 & 31 & & & 5 & 31 & & \\
\hline Distal & 8 & 56 & & & 7 & 57 & & & 6 & 58 & & \\
\hline Size & & & 0.227 & 0.633 & & & 0.156 & 0.693 & & & 0.484 & 0.487 \\
\hline$<5 \mathrm{~cm}$ & 5 & 45 & & & 6 & 44 & & & 6 & 44 & & \\
\hline$\geq 5 \mathrm{~cm}$ & 8 & 54 & & & 6 & 56 & & & 5 & 57 & & \\
\hline Lauren classification & & & 0.56 & 0.45 & & & 0.55 & 0.46 & & & 0.037 & 0.85 \\
\hline Intestinal & 8 & 50 & & & 5 & 53 & & & 6 & 52 & & \\
\hline Diffuse & 5 & 49 & & & 7 & 47 & & & 5 & 49 & & \\
\hline Histology & & & 1.53 & 0.675 & & & 5.376 & 0.146 & & & 2.62 & 0.454 \\
\hline Papillary adenocarcinoma & 0 & 5 & & & 0 & 5 & & & 0 & 5 & & \\
\hline Tubular adenocarcinoma & 11 & 69 & & & 12 & 68 & & & 10 & 70 & & \\
\hline Mucinous adenocarcinoma & 1 & 15 & & & 0 & 16 & & & 1 & 15 & & \\
\hline Signet-ring cell carcinoma & 1 & 10 & & & 0 & 11 & & & 0 & 11 & & \\
\hline Histologic differentiation & & & 0.013 & 0.91 & & & 1.97 & 0.16 & & & 0.10 & 0.75 \\
\hline Well-moderately & 4 & 32 & & & 6 & 30 & & & 4 & 32 & & \\
\hline Poorly-undifferentiated & 9 & 67 & & & 6 & 70 & & & 7 & 69 & & \\
\hline TNM Stages & & & 24.31 & 0.001 & & & 36.46 & 0.001 & & & 20.26 & 0.001 \\
\hline 1 & 7 & 7 & & & 8 & 6 & & & 6 & 8 & & \\
\hline$\|$ & 4 & 36 & & & 2 & 38 & & & 3 & 37 & & \\
\hline III & 2 & 37 & & & 2 & 37 & & & 1 & 38 & & \\
\hline IV & 0 & 19 & & & 0 & 19 & & & 1 & 18 & & \\
\hline Lymph node metastasis & & & 48.37 & 0.001 & & & 24.15 & 0.001 & & & 41.32 & 0.001 \\
\hline No & 7 & 1 & & & 5 & 3 & & & 6 & 2 & & \\
\hline Yes & 6 & 98 & & & 7 & 97 & & & 5 & 99 & & \\
\hline Distant metastasis & & & 0.898 & 0.343 & & & 2.746 & 0.098 & & & 0.537 & 0.464 \\
\hline No & 12 & 81 & & & 12 & 81 & & & 10 & 83 & & \\
\hline Yes & 1 & 18 & & & 0 & 19 & & & 1 & 18 & & \\
\hline
\end{tabular}

\section{Discussion}

The outcome of patients with gastric cancer depends on tumor stage at the time of diagnosis [13]. Endoscopic examination is the most reliable method for the diagnosis of gastric cancer; however, the feasibility and cost effectiveness of this invasive approach in most countries remain questionable [14]. A simple diagnostic test, such as a serum biomarker assay, could facilitate screening for gastric cancer. Currently, however, there are no serum biomarkers that are sufficiently sensitive and specific for the routine diagnosis of gastric cancer. The sensitivities of tumor markers such as CEA, CA 19-9 and CA 72-4 are low (20 to 30\%) [15-17]. O'Sullivan et al. [18] suggested that preoperative detection of micrometastases may reflect either transient shedding of cells, metastatic potential or residual disease.

In this study, we detected the expression levels of KAP1, TIMP1, STC2, TLN2, SRPX2 and SPARC mRNAs in peripheral blood samples from pre-operative gastric cancer patients, those with recurrence, and in healthy volunteers. ROC curve analysis was used to evaluate the diagnostic value of the expression levels of these genes 


\section{ROC Curve}

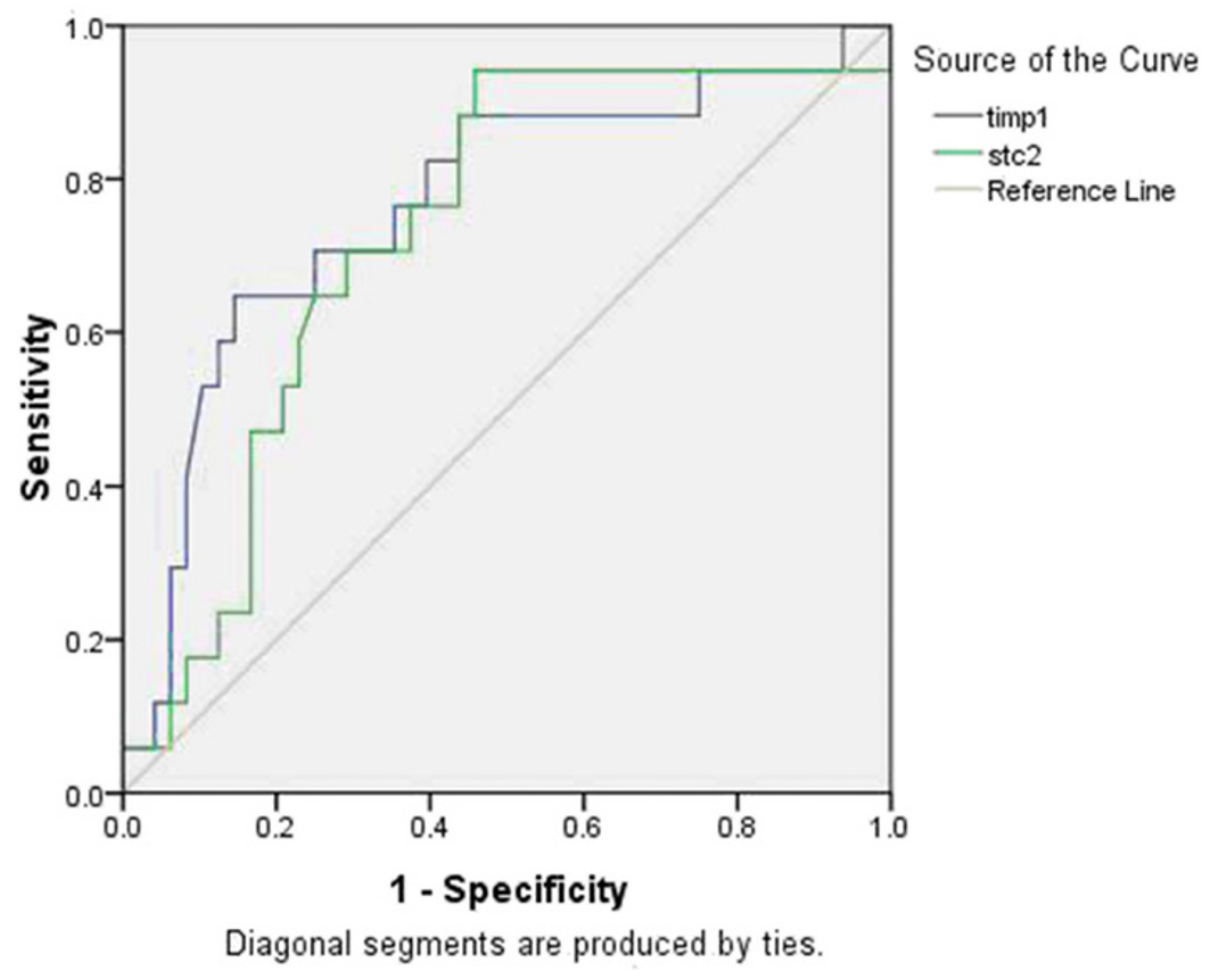

Figure 3 Receiver operation characteristics (ROC) curve of the TIMP1 and STC2 in peripheral blood of patients with recurrence of gastric cancer and healthy volunteers. The clinical values were assessed by 42 patients with recurrence of gastric cancer from 107 healthy volunteers.

in differentiating preoperative gastric cancer patients or patients with recurrence from healthy volunteers. The study showed that KAP1, TIMP1 and STC2 expression levels could differentiate preoperative gastric cancer patients from healthy volunteers, and that TIMP1 and STC2 could differentiate preoperative gastric cancer patients with recurrence from healthy volunteers.

Previous studies showed that TIMP1 was overexpressed in both liver and peritoneal metastases from patients with colorectal adenocarcinoma, melanoma and malignant thyroid neoplasms. TIMP1 showed significant increase in immunoreactivity in the colorectal carcinomatous epithelium compared with the adenomatous epithelium [19]. TIMP1 was overexpressed in both liver and peritoneal metastases from patients with colorectal adenocarcinoma [20]). The level of TIMP1 mRNA expression was found to be an independent diagnostic marker of malignant thyroid neoplasms [21]. In our study, the expression levels of STC2 in patients with gastric cancer were significantly higher than those in healthy volunteers. There were significant correlations between the expression levels of STC2, and TNM stages and lymph node metastasis. The level of serum TIMP-1 in patients with melanoma has previously been shown to be significantly higher than in controls, leading to the speculation that serum level of TIMP-1 may be a new useful marker for melanoma progression [22].

Solid tumor progression is usually associated with hypoxia. STC2 has been suggested as a novel target of oxidative stress response to protect cells from apoptosis. The expression of STC2 has been reported to be highly correlated with human cancer development. The identification and functional analysis of STC2 upregulation by hypoxia, a feature of the tumor microenvironment, sheds light on a possible role for STC2 in tumors. The cell proliferation was reduced in STC2-silenced cells but was increased in STC2-overexpressing hypoxic cells [23]. The stable expression of exogenous STC2 promoted epithelial-mesenchymal transition in hypoxic conditions. When STC2 was stably transfected into cells, they showed a high degree of motility with fibroblast morphology under hypoxic condition, and a high degree of invasiveness in hypoxia. The finding provides evidence that $S T C 2$ is a positive regulator of tumor progression in hypoxic conditions [24]. In our study, the expression of STC2 in patients with gastric cancer was significantly higher than in healthy volunteers. There were significant correlations between the expression of STC2, and TNM 
stages and lymph node metastasis. Previous studies have shown that the expression of STC2 in colorectal cancer tissue was higher than in corresponding normal colorectal epithelial tissue. The high expression of STC2 was correlated with lymph node metastasis, lymphatic invasion, tumor depth, tumor size, AJCC stage classification and worse overall survival rates [9]. STC2 was upregulated at the mRNA and protein levels in renal cell carcinoma [25]. STC2 was expressed in invasive breast tumor cells, while univariate survival analysis revealed that expressions of STC2 were correlated with longer disease-free survival [26].

In our study, we focused on the KAP1 expression patterns in peripheral blood from patients with gastric cancer. The levels of $K A P 1$ in the gastric cancer group were found to be significantly higher than those in the healthy volunteer group. There were significant correlations between the expression of $K A P 1$, and TNM stages and distant metastasis. The study by Yokoe et al. showed that the expression of KAP1 was significantly higher in cancerous tissues than in non-cancerous tissues. Patients with high KAP1 expression showed a higher incidence of peritoneal carcinomatosis and significantly poorer overall survival compared with patients with low KAP1 expression. Multivariate analysis revealed that high $K A P 1$ expression was an independent prognostic factor. Intriguingly, high $K A P 1$ expression was also an independent factor for peritoneal carcinomatosis [10]. To evaluate the diagnostic value of KAP1 in the diagnosis of patients with recurrence of gastric cancer, the AUC value from ROC curve analysis was determined. The AUC of KAP1 was $0.418 \pm 0.088(P=0.319)$, indicating that expression levels of KAP1 could not distinguish recurrence of gastric cancer from cancer, probably because the number of recurrences of gastric cancer were too few. The proliferation rate was impaired and resistance to anoikis was decreased after knockdown of KAP1 in the gastric cancer cell lines AZ521 and KATO III [10]. $K A P 1$ contributes to the negative regulation of E2F1 and may serve as a partial backup to prevent E2F1-mediated apoptosis in the absence of pRb [27].

Previous studies have shown that SRPX2 and SPARC were overexpressed in gastric cancer tissues [28-30], while TLN2 was upregulated in breast carcinomas tissues [31]; however, no studies investigating the expression of TLN2, SRPX2, and SPARC in peripheral blood have been reported. Our study showed that TLN2, $S R P X 2$ and SPARC expression were detected in gastric cancer, but expression of TLN2, SRPX2 and SPARC could not differentiate preoperative gastric cancer patients from healthy volunteers.

KAP1, TIMP1 and STC2 appear to have a role in the progression to metastatic disease in gastric cancer. Preoperative KAP1, TIMP1 and STC2 expression levels in peripheral blood were related to cancer stage and may be markers of tumor invasion, lymph node metastasis and TNM stage. In particular, high TIMP1 and STC2 expression levels could be poor prognostic factors of disease recurrence in patients with gastric cancer.

\section{Conclusions}

Expression levels of KAP1, TIMP1 and STC2 are potentially useful as clinical biomarkers for the screening, diagnosis, prognostic and surveillance of gastric cancer.

\section{Abbreviations}

AJCC: American Joint Committee on Cancer; AUC: Area under the ROC curves; bp: Base pairs; EMT: Epithelial-mesenchymal transition; ITGB1: Integrin beta 1; KAP1: Kinesin II-associated protein; MMPs: Matrix metalloproteinases; ROC: Receiver operation characteristics; SPARC: Secreted protein, acidic, cysteine-rich; SRPX2: Sushi-repeat-containing protein, X-linked 2;

STC2: Stanniocalcin 2; TIMP: Tissue inhibitor of metalloproteases; TIMP1: TIMP metallopeptidase inhibitor 1; TLN2: Talin 2.

\section{Competing interests}

We declare that we have no conflicts of interest.

\section{Authors' contributions}

Y-YW, LL, and H-JW participated in the scientific experiments. All authors participated in the conception and design of the study, as well as data collection and interpretation, manuscript preparation and literature search. All authors have read and approved the final manuscript.

\section{Acknowledgements}

Work was supported by Zhejiang Provincial Department of Science and Technology Research Foundation (2008C33040).

\section{Author details}

'Department of Gastrointestinal Surgery, Zhejiang Provincial People's Hospital, 158 shangtang road, Hangzhou, Zhejiang, PR China310014. ${ }^{2}$ Department of Pathology, Zhejiang Provincial People's Hospital, 158 shangtang road, Hangzhou, Zhejiang, PR China310014. ${ }^{3}$ Key Laboratory of Gastroenterology of Zhejiang Province, 158 shangtang road, Hangzhou, Zhejiang, PR China310014.

Received: 28 September 2012 Accepted: 9 March 2013 Published: 2 April 2013

\section{References}

1. Zhan $\mathrm{WH}, \mathrm{Han} \mathrm{FH}$ : Surgical therapy of gastric cancer in china. J Pract Oncol 2008, 23:91-93.

2. Ghossein RA, Rosai J: Polymerase chain reaction in the detection of micrometastases and circulating tumor cells. Cancer 1996, 78:10-16.

3. Pelkey TJ, Frierson HF Jr, Bruns DE: Molecular and immunological detection of circulating tumor cells and micrometastases from solid tumors. Clin Chem 1996, 42:1369-1381.

4. Marrelli D, Roviello F, De Stefano A, Farnetani M, Garosi L, Messano A, Pinto E: Prognostic significance of CEA, CA 19-9 and CA 72-4 preoperative serum levels in gastric carcinoma. Oncology 1999, 57:55-62.

5. Holten-Andersen MN, Hansen U, Brünner N, Nielsen HJ, Illemann M, Nielsen BS: Localization of tissue inhibitor of metalloproteinases 1 (TIMP-1) in human colorectal adenoma and adenocarcinoma. Int J Cancer 2005 113:198-206.

6. Bommarito A, Richiusa P, Carissimi E, Pizzolanti G, Rodolico V, Zito G, Criscimanna A, Di Blasi F, Pitrone M, Zerilli M, Amato MC, Spinelli G, Carina V, Modica G, Latteri MA, Galluzzo A, Giordano C: BRAFV600E mutation, TIMP-1 upregulation, and NF-KB activation: closing the loop on the papillary thyroid cancer trilogy. Endocr Relat Cancer 2011, 18:669-685.

7. Raulic S, Ramos-Valdes Y, DiMattia GE: Stanniocalcin 2 expression is regulated by hormone signalling and negatively affects breast cancer cell viability in vitro. J Endocrinol 2008, 197:517-529.

8. Kita Y, Mimori K, Iwatsuki M, Yokobori T, leta K, Tanaka F, Ishii H, Okumura H, Natsugoe S, Mori M: STC2: a predictive marker for lymph node metastasis 
in esophageal squamous-cell carcinoma. Ann Surg Oncol 2011, 18:261-272

9. leta K, Tanaka F, Yokobori T, Kita Y, Haraguchi N, Mimori K, Kato H, Asao T, Inoue $\mathrm{H}$, Kuwano H, Mori M: Clinicopathological significance of stanniocalcin 2 gene expression in colorectal cancer. Int J Cancer 2009, 125:926-931.

10. Yokoe T, Toiyama Y, Okugawa Y, Tanaka K, Ohi M, Inoue Y, Mohri Y, Miki C, Kusunoki M: KAP1 is associated with peritoneal carcinomatosis in gastric cancer. Ann Surg Oncol 2010, 17:821-828.

11. Wang YY, Ye ZY, Zhao ZS, Tao HQ, Chu YQ: High-level expression of S100A4 correlates with lymph node metastasis and poor prognosis in patients with gastric cancer. Ann Surg Oncol 2010, 17:89-97.

12. Tsujiura M, Ichikawa D, Komatsu S, Shiozaki A, Takeshita H, Kosuga T, Konishi H, Morimura R, Deguchi K, Fujiwara H, Okamoto K, Otsuji E: Circulating microRNAs in plasma of patients with gastric cancers. Br J Cancer 2010, 102:1174-1179.

13. Jemal A, Siegel R, Ward E, Murray T, Xu J, Thun MJ: Cancer statistics, 2007. CA Cancer J Clin 2007, 57:43-66.

14. Genta RM: Screening for gastric cancer: does it make sense? Aliment Pharmacol Ther 2004, 20:42-47.

15. Marrelli D, Pinto E, De Stefano A, Farnetani M, Garosi L, Roviello F: Clinical utility of CEA, CA 19-9, and CA 72-4 in the follow-up of patients with resectable gastric cancer. Am J Surg 2001, 181:16-19.

16. Ishigami S, Natsugoe S, Hokita S, Che X, Tokuda K, Nakajo A, Iwashige H, Tokushige M, Watanabe T, Takao S, Aikou T: Clinical importance of preoperative carcinoembryonic antigen and carbohydrate antigen 19-9 levels in gastric cancer. J Clin Gastroenterol 2001, 32:41-44

17. Gaspar MJ, Arribas I, Coca MC, Díez-Alonso M: Prognostic value of carcinoembryonic antigen, CA 19-9 and CA 72-4 in gastric carcinoma. Tumour Biol 2001, 22:318-322.

18. O'Sullivan GC, Collins JK, Kelly J, Morgan J, Madden M, Shanahan F: Micrometastases: marker of metastatic potential or evidence of residual disease? Gut 1997, 40:512-515.

19. Jeffery N, McLean MH, El-Omar EM, Murray Gl: The matrix metalloproteinase/tissue inhibitor of matrix metalloproteinase profile in colorectal polyp cancers. Histopathology 2009, 54:820-828.

20. Varghese $S$, Burness $M, X u H$, Beresnev T, Pingpank J, Alexander HR: Site-specific gene expression profiles and novel molecular prognostic factors in patients with lower gastrointestinal adenocarcinoma diffusely metastatic to liver or peritoneum. Ann Surg Oncol 2007, 14:3460-3471

21. Kebebew E, Peng M, Reiff E, McMillan A: Diagnostic and extent of disease multigene assay for malignant thyroid neoplasms. Cancer 2006, 106:2592-2597.

22. Yoshino Y, Kageshita T, Nakajima M, Funakubo M, Ihn H: Clinical relevance of serum levels of matrix metallopeptidase-2, and tissue inhibitor of metalloproteinase- 1 and -2 in patients with malignant melanoma. J Dermatol 2008, 35:206-214.

23. Law AY, Wong CK: Stanniocalcin-2 is a HIF-1 target gene that promotes cell proliferation in hypoxia. Exp Cell Res 2010, 316:466-476.

24. Law AY, Wong CK: Stanniocalcin-2 promotes epithelial-mesenchymal transition and invasiveness in hypoxic human ovarian cancer cells. Exp Cell Res 2010, 316:3425-3434.

25. Meyer HA, Tölle A, Jung M, Fritzsche FR, Haendler B, Kristiansen I, Gaspert A, Johannsen M, Jung K, Kristiansen G: Identification of stanniocalcin 2 as prognostic marker in renal cell carcinoma. Eur Urol 2009, 55:669-678.

26. Esseghir S, Kennedy A, Seedhar P, Nerurkar A, Poulsom R, Reis-Filho JS, Isacke CM: Identification of NTN4, TRA1, and STC2 as prognostic markers in breast cancer in a screen for signal sequence encoding proteins. Clin Cancer Res 2007, 13:3164-3173.

27. Wang C, Rauscher FJ 3rd, Cress WD, Chen J: Regulation of E2F1 function by the nuclear corepressor KAP1. J Biol Chem 2007, 282:29902-29909.

28. Tanaka K, Arao T, Maegawa M, Matsumoto K, Kaneda H, Kudo K, Fujita Y, Yokote H, Yanagihara K, Yamada Y, Okamoto I, Nakagawa K, Nishio K: SRPX2 is overexpressed in gastric cancer and promotes cellular migration and adhesion. Int J Cancer 2009, 124:1072-1080.

29. Wang L, Yang M, Shan L, Qi L, Chai C, Zhou Q, Yao K, Wu H, Sun W: The role of SPARC protein expression in the progress of gastric cancer. Pathol Oncol Res 2012, 18:697-702.
30. Zhao ZS, Wang YY, Chu YQ, Ye ZY, Tao HQ: SPARC is associated with gastric cancer progression and poor survival of patients. Clin Cancer Res 2010, 16:260-268.

31. Haakensen VD, Bjøro T, Lüders T, Riis M, Bukholm IK, Kristensen VN, Troester MA, Homen MM, Ursin G, Børresen-Dale AL, Helland A: Serum estradiol levels associated with specific gene expression patterns in normal breast tissue and in breast carcinomas. BMC Cancer 2011, 11:332.

doi:10.1186/1477-7819-11-8

Cite this article as: Wang et al: Clinical utility of measuring expression levels of KAP1, TIMP1 and STC2 in peripheral blood of patients with gastric cancer. World Journal of Surgical Oncology 2013 11:81.

\section{Submit your next manuscript to BioMed Central and take full advantage of:}

- Convenient online submission

- Thorough peer review

- No space constraints or color figure charges

- Immediate publication on acceptance

- Inclusion in PubMed, CAS, Scopus and Google Scholar

- Research which is freely available for redistribution 